\title{
Relatório de pareceristas da Revista de Sociologia e Política em 2012
}

Board of Reviewers of Revista de Sociologia e Política in 2012 Relación de evaluadores de la Revista de Sociologia e Política en 2012

Adriana Marques Rossetto (UFSC) ad hoc

Adriano Oliveira (UFPE) ad hoc

Ailton Souza (UEMS) ad hoc

Alan Daniel Freire de Lacerda (UFRN) ad hoc

Aldo Duran Gil (UFU) ad hoc

Alexandre Pereira da Rocha (UnB) ad hoc

Alexandre Queiroz Guimarães (PUC-MG) ad hoc

Alexsandro Eugênio Pereira (UFPR)

Ana Cláudia Chaves Teixeira (Unicamp) ad hoc

Ana Lúcia de Sousa (UFRR) ad hoc

Ana Luiza d'Ávila Viana (USP) ad hoc

Ana Luiza Melo Aranha (UFMG) ad hoc

Ana Maria Rita Milani (UFAL) ad hoc

André Borges (UnB) ad hoc

André Luiz Reis da Silva (UFRGS) ad hoc

André Silva (USP) ad hoc

Andréa Cristina Oliveira Gozetto (Uninove) ad hoc

Andréa Freitas (USP) ad hoc

Andreia Reis do Carmo (UnB) ad hoc

Andressa Silvério Terra França (UFPR) ad hoc

Ângela Randolpho Paiva (PUC-RJ) ad hoc

Aníbal Jáuregui (UBA)

Antônia de Lourdes Colbari (UFES) ad hoc

Antônio Carlos Moraes Lessa (UnB) ad hoc

Arnaldo Provasi Lanzara (UERJ) ad hoc

Ary Cesar Minella (UFSC)

Augusto Junior Clemente (UFRGS) ad hoc

Bárbara Lamas (UERJ) ad hoc

Bruna Gisi (USP)

Bruno Bolognesi (Ufscar) ad hoc

Bruno Vicente Lippe Pasquarelli (Ufscar) ad hoc

Camilo Aggio (UFBA) ad hoc

Carla Almeida (UEM) ad hoc

Carlos A. Gadea (Unisinos) ad hoc

Carlos Aurélio Pimenta de Faria (PUC-MG) ad hoc

Carlos Eduardo Sell (UFSC) ad hoc

Carlos Pinho (UERJ) ad hoc

Carlos Vasconcelos Rocha (PUC-MG) ad hoc

Celene Tonella (UEM) ad hoc

César Augusto Silva da Silva (UFGD) ad hoc

Christian Edward Cyril Lynch (Unirio) ad hoc

Cintia Russo (Universidad Nacional de Quilmes, Argentina) ad hoc

Clara Maria Farias de Araujo (UFRJ) ad hoc

Cláudia Cristina Azeredo Atallah (UFF) ad hoc

Cláudio Luis de Camargo Penteado (Ufabc) ad hoc

Cloves Barbosa (UFPA) ad hoc

Clóvis Roberto Zimmermann (UFRB) ad hoc 
Corival Alves do Carmo (UFSE) ad hoc

Cristiane Correa Batista Santos (Unirio) ad hoc

Cristiano Fonseca Monteiro (UFF) ad hoc

Cristiano Luis Lenzi (USP) ad hoc

Cristina Monteiro de Queiroz (UnB) ad hoc

Cristina Soreanu Pecequilo (Unesp) ad hoc

Daniel de Mendonça (Ufpel) ad hoc

Daniel Soczek (Uninter) ad hoc

Danilo Rothberg (Unesp) ad hoc

Dawisson Belém Lopes (UFMG) ad hoc

Débora Cristina Rezende de Almeida (UnB) ad hoc

Déborah Barros Leal Farias (University of British Columbia) ad hoc

Denise Paiva Ferreira (UFG) ad hoc

Diego Santos Vieira de Jesus (PUC-RJ) ad hoc

Dina Lida Kinoshita (USP; Unesco) ad hoc

Doacir Gonçalves de Quadros (Uninter) ad hoc

Domitila Castro Cayres (UFSC) ad hoc

Dulcinéa Duarte de Medeiros (UFF) ad hoc

Edir Veiga Siqueira (UFPA) ad hoc

Ednaldo Ribeiro (UEM) ad hoc

Eduardo Lopes Cabral Maia (UFSM) ad hoc

Eduardo R. Gomes (UFF) ad hoc

Eli Diniz (UFRJ)

Elsio Lenardão (UEL) ad hoc

Emerson Urizzi Cervi (UFPR)

Euzeneia Carlos (UFES) ad hoc

Fábia Berlatto (UFPR) ad hoc

Fabiano Santos (UERJ) ad hoc

Fabio Kobol Fornazari (MinC) ad hoc

Fabrício Mazocco (Ufscar) ad hoc

Feliciano de Sá Guimarães (ESPM) ad hoc

Felipe Calabrez da Silva (UFPR) ad hoc

Felipe de Moraes Borba (Unirio) ad hoc

Felipe Nunes dos Santos (UCLA, Estados Unidos) ad hoc

Felix Garcia Lopez (IPEA) ad hoc

Fernando Azevedo (Ufscar) ad hoc

Fernando Baptista Leite (UFPR) ad hoc

Fernando Coelho (USP) ad hoc

Fernando de Barros Filgueiras (UFMG) ad hoc

Fernando Perlatto Bom Jardim (UERJ) ad hoc

Flávia Biroli (UnB) ad hoc

Flávio Ramos (Univali) ad hoc

Francisco Jatobá de Andrade (USP) ad hoc

Gabriel Cepaluni (Unesp) ad hoc

Gabriel Eduardo Vitullo (UFRN) ad hoc

Gelsom Rozentino de Almeida (UERJ) ad hoc

George Avelino Filho (Fundação Getúlio Vargas) ad hoc

Glauco Avelino Sampaio Oliveira (Ministério da Fazenda) ad hoc

Graciela Zubelzú de Bacigalupo (Universidad Nacional de Rosário, Argentina)

Guilherme Radomsky (UFRGS) ad hoc

Gustavo Biasoli Alves (Unioeste) ad hoc

Gustavo Biscaia de Lacerda (UFPR)

Hilton Costa (UFPR) ad hoc

Hugo Borsani (UENF) ad hoc

Ignacio Godinho Delgado (UFJF) ad hoc 
Igor Ferraz da Fonseca (IPEA) ad hoc

Ivann Carlos Lago (UFFS) ad hoc

Ivo Coser (UFRJ) ad hoc

Jamila Rainha (UFES) ad hoc

Javier Amadeo (Unifesp) ad hoc

Javier Vadell (PUC-MG) ad hoc

Joana Tereza Vaz de Moura (UFRN) ad hoc

João Carlos Amoroso Botelho (UFG) ad hoc

Jorge Zaverucha (UFPE) ad hoc

José Cláudio Berghella (Ufscar) ad hoc

José Maria Nóbrega Jr. (UFCG) ad hoc

Juan Claudio Epsteyn (Ibmec-RJ) ad hoc

Juarez Varallo Pont (UFPR) ad hoc

Júlia Bertino Moreira (Unicamp) ad hoc

Kátia Sento Sé Mello (UFRJ) ad hoc

Kelly Cristina Costa Soares (UFCG) ad hoc

Klarissa Almeida Silva (UFRJ) ad hoc

Leonardo Biscaia de Lacerda (UFPR) ad hoc

Letícia M. Schabbach (UFRGS) ad hoc

Lígia Helena Hahn Lüchmann (UFSC) ad hoc

Lorena Madruga Monteiro (UFRGS) ad hoc

Lucas Massimo (Unicamp)

Lucas Rodrigues Cunha (IPEA) ad hoc

Luciana Leite Lima (UFRGS) ad hoc

Luciano Da Ros (UFRGS) ad hoc

Lúcio Rennó (UnB) ad hoc

Ludmila Mendonça Lopes Ribeiro (UFMG) ad hoc

Luis Felipe Miguel (UnB)

Luis Fernando Ayerbe (Unesp) ad hoc

Luiz Daniel Jatobá França (UnB) ad hoc

Luiz Felipe Brandão Osório (UFRJ) ad hoc

Luiz Paulo Rouanet (UFSJ) ad hoc

Marcelo da Silveira Campos (USP) ad hoc

Marcelo Martins Barreira (UFES) ad hoc

Marcia da Silva Mazon (UFSC) ad hoc

Marco Cepik (UFRGS)

Marcos Silva da Silveira (UFPR) ad hoc

Maria Alejandra Nicolás (UFPR) ad hoc

Maria Clara de Arruda Barbosa (UERJ) ad hoc

Mariana Pfeiffer (UFF) ad hoc

Mariele Troiano (Ufscar) ad hoc

Marília Patta Ramos (UFRGS) ad hoc

Marta Fernandez Moreno (PUC-RJ) ad hoc

Matheus de Carvalho Hernandez (UFGD) ad hoc

Maurício Izumi (USP) ad hoc

Maurício Santoro (FGV) ad hoc

Miguel Serna (Udelar)

Nelson Rosário de Souza (UFPR)

Paolo Ricci (USP) ad hoc

Patrícia Duarte Rangel (PUC-GO) ad hoc

Paulo G. Fagundes Vizentini (UFRGS) ad hoc

Paulo Henrique Canhoto Alves (UDF) ad hoc

Paulo Peres (UFRGS) ad hoc

Pedro Santos Mundim (UFG) ad hoc

Rafael Assumpção de Abreu (UERJ) ad hoc 
Rafael Cardoso Sampaio (UFBA) ad hoc

Rafael Machado Madeira (PUC-RS) ad hoc

Raquel Andrade Weiss (Ufrgs) ad hoc

Raúl Burgos (UFSC) ad hoc

Raul Francisco Magalhães (UFJF) ad hoc

Rebecca Abbers (UnB) ad hoc

Renan Springer de Freitas (UFMG) ad hoc

Renata Bichir (Ministério do Desenvolvimento Social) ad hoc

Renato Monseff Perissinotto (UFPR)

Ricardo Borges Gama Neto (UFPE) ad hoc

Ricardo Carneiro (Fundação João Pinheiro) $a d$ hoc

Ricardo Fabrino de Medonça (UFMG) ad hoc

Rodrigo Rossi Horochovski (UFPR) ad hoc

Rogério Bezerra da Silva (Unicamp) ad hoc

Rosane Cristina de Oliveira (UERJ) ad hoc

Rossana Rocha Reis (USP) ad hoc

Samira Kauchakje (PUC-PR) ad hoc

Sandra Cristina Gomes (UFRN) ad hoc

Sandro Pereira Silva (IPEA) ad hoc

Sérgio Praça (Fundação Getúlio Vargas) ad hoc

Simone Diniz (Ufabc) ad hoc

Simone Rodrigues Bohn (York University, Canadá) ad hoc

Sydenham Lourenço Neto (UERJ) ad hoc

Terezinha Richartz Santana (Centro Universitário do Sul de Minas Gerais) ad hoc

Thales Novaes de Andrade (Ufscar) ad hoc

Thiago Lima (Cedec) ad hoc

Thiago Rodrigues Silame (UFMG) ad hoc

Tiago Bahia Losso (UFSC) ad hoc

Torcuato Di Tella (Instituto Di Tella, Argentina) ad hoc

Vanessa Elias de Oliveira (Ufabc) ad hoc

Vanessa Empinotti (USP) ad hoc

Vilma Soares de Lima Barbosa (UECG) ad hoc

Vilson da Mata (UFPR) ad hoc

Vitor Marchetti (Ufabc) ad hoc

Viviana Román (Universidad de Buenos Aires, Argentina) ad hoc

Viviani Corrêa Teixeira (UFSC) ad hoc

Wagner de Melo Romão (Unesp) ad hoc

Wagner Iglécias (USP) ad hoc

Wagner Pralon Mancuso (USP) ad hoc

Williams da Silva Gonçalves (UERJ) ad hoc 